\title{
Reconstructing the right-hand side of a fractional subdiffusion equation from the final data
}

\author{
Nguyen Hoang Luc ${ }^{1}$, Dumitru Baleanu ${ }^{2,3,4}$, Le Dinh Long ${ }^{5,6}$ and Nguyen-Huu Can ${ }^{7 *}$ (1)
}

\author{
"Correspondence: \\ nguyenhuucan@tdtu.edu.vn \\ ${ }^{7}$ Applied Analysis Research Group, \\ Faculty of Mathematics and \\ Statistics, Ton Duc Thang University, \\ Ho Chi Minh City, Vietnam \\ Full list of author information is \\ available at the end of the article
}

\begin{abstract}
In this study, we study an inverse source problem for the time-fractional diffusion equation, where the final data $t=T$ are given. We show that our problem is ill-posed in the sense of Hadamard. Applying a truncation method, we give the regularized solution. Finally, convergence estimates under a priori and a posteriori parameter choice rules are proved.
\end{abstract}

MSC: $35 \mathrm{~K} 05 ; 35 \mathrm{~K} 99 ; 47 \mathrm{J06} ; 47 \mathrm{H} 10$

Keywords: Time-fractional diffusion equation; Truncation method; Inverse source problem

\section{Introduction}

The last few decades have seen interest in the research of diffusion equation problems; more and more researchers are studying the problem of the fractional diffusion equation. It can model anomalous phenomena in chemical physics, biological cell dynamics, physiology, and finance [1-4]. In this study, we consider the following fractional diffusion equation with Riemann-Liouville derivative:

$$
\left\{\begin{array}{l}
\partial_{t} u(x, y, t)=\partial_{t}^{1-\beta}\left(u_{x x}(x, y, t)+u_{y y}(x, y, t)\right)+\Phi(t) f(x, y) \\
\quad(x, y, t) \in \Omega \times(0, T) \\
u(x, y, t)=0, \quad(x, y) \in \partial \Omega, t \in(0, T] \\
u(x, y, T)=g(x, y), \quad(x, y) \in \Omega
\end{array}\right.
$$

with the Riemann-Liouville fractional derivative of order $1-\beta \in(0,1)$ defined by $[3,5]$ as follows:

$$
\partial_{t}^{1-\beta} u(x, y, t)=\frac{1}{\Gamma(\beta)} \frac{d}{d t} \int_{0}^{t}(t-\varsigma)^{\beta-1} u(x, y, \varsigma) d \varsigma, \quad t>0
$$

where $\Gamma(\cdot)$ is the Gamma function and $\Omega=(0, \pi)^{2}$. The Laplacian operator $\Delta u(x, y, t)=$ $u_{x x}(x, y, t)+u_{y y}(x, y, t)$ (with the homogeneous Dirichlet boundary condition) has the nor-

(c) The Author(s) 2020. This article is licensed under a Creative Commons Attribution 4.0 International License, which permits use, sharing, adaptation, distribution and reproduction in any medium or format, as long as you give appropriate credit to the original author(s) and the source, provide a link to the Creative Commons licence, and indicate if changes were made. The images or other third party material in this article are included in the article's Creative Commons licence, unless indicated otherwise in a credit line to the material. If material is not included in the article's Creative Commons licence and your intended use is not permitted by statutory regulation or exceeds the permitted use, you will need to obtain permission directly from the copyright holder. To view a copy of this licence, visit http://creativecommons.org/licenses/by/4.0/. 
malized eigenbasis

$$
e_{k}(x)=\left(\frac{2}{\pi}\right)^{\frac{d}{2}} \sin \left(k_{1} x_{1}\right) \cdots \sin \left(k_{d} x_{d}\right) \in \mathbb{R}^{d}
$$

and the eigenvalues $\left(p^{2}+q^{2}\right)$. We denote $p=\left(p_{1}, p_{2}, \ldots, p_{d}\right), x=\left(x_{1}, x_{2}, \ldots, x_{d}\right)$ and $p^{2}=$ $p_{1}^{2}+\cdots+p_{d}^{2}$. Similarly, we have $q=\left(q_{1}, q_{2}, \ldots, q_{d}\right), y=\left(y_{1}, y_{2}, \ldots, y_{d}\right)$ and $q^{2}=q_{1}^{2}+\cdots+q_{d}^{2}$. Problem (1.1) has been studied by many authors. The direct problem (or the initial value problem) concerning the first equation of (1.1) seems to come from [6] where the authors introduced the integral formulation of the fractional diffusion equation. These equations have attracted a lot of attention, see, e.g., [7, 8]. Models with a singular kernel at the origin are well-known and arise in the heat conduction and viscoelasticity, etc. [9, 10]. Some works for the linear case can be found in $[9,10]$. When we know the final data $g$, the problem (1.1) is about recovering the initial data $u(x, y, 0)$, called the backward problem, which was studied recently by Yong et al. [11]. Our main aim in this paper is to recover the source function $f$ from the given data $g(x, y)$ and $\Phi(t)$. Here $f(x, y)$ and $\Phi(t)$ describe the spatial distribution of the source and the time evolution pattern, respectively. In this case, our problem is called the inverse source problem (ISP) which is motivated by many practical applications. We can list some applications of (ISP), for example, pollution in the environment [12, 13], dislocation problems [14], biomedical imaging techniques such as the thermo-acoustic tomography $[15,16]$, electroencephalography/magnetoencephalography (EEG/MEG) problems [17, 18], optical tomography [19]. The inverse source problem for some other fractional diffusion equations has been investigated by many authors and its physical background can be found in [20]; see also the works by Wei et al. [21-23] and Kirane et al. [24, 25]. To the best of our knowledge, there are no results for the inverse source problem (1.1).

Now, we return and discuss more details on the purpose of our paper. It is known that the inverse source problem mentioned above is not well-posed in general, i.e., when a solution exists, it does not depend continuously on the given data. In practice, the exact data $(\Phi, g)$ is noised by measured data $\left(\Phi^{\varepsilon}, g^{\varepsilon}\right)$ with order of $\varepsilon>0$ as follows:

$$
\left\|\Phi^{\varepsilon}-\Phi\right\|_{\mathcal{L}^{\infty}(0, T)} \leq \varepsilon, \quad\left\|g^{\varepsilon}-g\right\|_{\mathcal{L}^{2}(\Omega)} \leq \varepsilon
$$

where $\|\Phi\|_{\mathcal{L}^{\infty}(0, T)}=\sup _{0 \leq t \leq T}|\Phi(t)|$ for any $\Phi \in \mathcal{L}^{\infty}(0, T)$. This problem is ill-posed in the sense of Hadamard, which means that small changes in the observed data can lead to a blow-up of the solution. Hence some regularization methods are required for stable computation of a sought solution. The topic of this paper is to find an approximate solution. Employing some previously suggested ideas, in this study, using the Fourier regularization method, we establish a regularized solution. Under an a priori bound assumption of the sought solution and a priori parameter choice rule, we give the convergence rate. In practice, an a priori bound is difficult to obtain and check, so we need an a posteriori parameter choice rule. The strong point of an a posteriori parameter choice rule is that it does not depend on the a priori bound.

The paper is organized into three sections. In Sect. 2, we present a formula of the source function $f$ and establish some lemmas and theorems which are useful to obtain the next results. The ill-posedness of the inverse source problem is also shown in this section. In 
Sect. 3, we apply the Fourier regularization method and give two convergence estimates and two regularization parameter choice rules: an a priori parameter choice and an $a$ posteriori parameter choice are presented in Sect. 3.1 and Sect. 3.2, respectively.

\section{Preliminary results}

Definition 2.1 Let $\langle\cdot, \cdot\rangle$ be a scalar product in $\mathcal{L}^{2}(\Omega)$. The notation $\|\cdot\|_{X}$ stands for the norm in the Banach space $X$. We denote by $\mathcal{L}^{p}(0, T ; X), 1 \leq p<\infty$, the Banach space of $p$-integrable real-valued functions $u:(0, T) \rightarrow X$, and set

$$
\|u\|_{\mathcal{L}^{\infty}(0, T ; X)}=\mathrm{ess} \sup _{t \in(0, T)}\|u(t)\|_{X}, \quad \text { for } p=\infty .
$$

Definition 2.2 (see [19]) The Mittag-Leffler function is defined by the series

$$
E_{\kappa, \alpha}(z)=\sum_{n=0}^{\infty} \frac{z^{n}}{\Gamma(\kappa n+\alpha)}, \quad \kappa>0, \alpha \in \mathbb{R}, z \in \mathbb{C}
$$

where $\kappa>0, \alpha \in \mathbb{R}$ are arbitrary constants.

Lemma 2.1 (see [19]) Let $\kappa>0$ and $\alpha \in \mathbb{R}$. Then one has

$$
E_{\kappa, \alpha}(z)=z E_{\kappa, \kappa+\alpha}(z)+\frac{1}{\Gamma(\alpha)}, \quad z \in \mathbb{C} .
$$

Lemma 2.2 (see [26]) Letting $\lambda>0$ and $\beta>0$ with $k$ being and integer from $\mathbb{N}^{*}$, one has

$$
\begin{aligned}
& \frac{d^{k}}{d t^{k}} E_{\beta, 1}\left(-\lambda t^{\beta}\right)=-\lambda t^{\beta-k} E_{\beta, \beta-k+1}\left(-\lambda t^{\beta}\right), \quad t>0, \\
& \frac{d}{d t}\left(t E_{\beta, 2}\left(-\lambda t^{\beta}\right)\right)=E_{\beta, 1}\left(-\lambda t^{\beta}\right), \quad t>0 .
\end{aligned}
$$

Lemma 2.3 (see [3]) Let $0<\beta_{0}<\beta_{1}<1$. Then there exist positive constants $\widetilde{\Lambda}_{1}, \widetilde{\Lambda}_{2}, \widetilde{\Lambda}_{3}$ depending only on $\beta_{0}, \beta_{1}$ such that for all $\beta \in\left[\beta_{0}, \beta_{1}\right]$,

$$
\frac{\tilde{\Lambda}_{1}}{1+z} \leq E_{\beta, 1}(-z) \leq \frac{\tilde{\Lambda}_{2}}{1+z}, \quad E_{\beta, \kappa}(-z) \leq \frac{\tilde{\Lambda}_{3}}{1+z}, \quad \text { for all } z \geq 0, \kappa \in \mathbb{R} .
$$

Lemma 2.4 (see [3]) Let $0<\beta<1$ and $\lambda, a>0$. Then

(i) $\partial_{t}\left(E_{\beta}\left(-\lambda t^{\beta}\right)\right)=-\lambda t^{\beta-1} E_{\beta, \beta}\left(-\lambda t^{\beta}\right)$, for $t \geq 0$,

(ii) $\partial_{t}\left(t^{\beta-1} E_{\beta, \beta}\left(-\lambda t^{\beta}\right)\right)=t^{\beta-2} E_{\beta, \beta-1}\left(-\lambda t^{\beta}\right)$, for $t \geq 0$,

(iii) $\int_{0}^{\infty} e^{-s t} E_{\alpha}\left(-a t^{\alpha}\right)=\frac{s^{\alpha-1}}{s^{\alpha}+a}$, for $\Re(s)>a^{\frac{1}{\alpha}}$.

Lemma 2.5 Let $0<\beta<1$, for $p, q>0$ and denote $\tilde{\lambda}_{p q}=p^{2}+q^{2}$, then

$$
\frac{\widetilde{\mathcal{M}}_{\beta}^{\dagger}\left(\tilde{\Lambda}_{1}\right)}{\widetilde{\lambda}_{p q}} \leq \int_{0}^{T} E_{\beta, 1}\left(-\widetilde{\lambda}_{p q}(T-\varsigma)^{\beta}\right) d \varsigma \leq \frac{\widetilde{\mathcal{M}}_{\beta}^{\dagger \dagger}\left(\widetilde{\Lambda}_{2}\right)}{\widetilde{\lambda}_{p q}},
$$

where

$$
\widetilde{M}_{\beta}^{\dagger}\left(\widetilde{\Lambda}_{1}\right)=\frac{2 \widetilde{\Lambda}_{1} T}{1+2 T^{\beta}}, \quad \widetilde{M}_{\beta}^{\dagger \dagger}\left(\widetilde{\Lambda}_{2}\right)=\frac{\widetilde{\Lambda}_{2} T^{1-\beta}}{1-\beta} .
$$


Proof From Lemma 2.3, we have

$$
\begin{aligned}
\int_{0}^{T} E_{\beta, 1}\left(-\tilde{\lambda}_{p q}(T-\varsigma)^{\beta}\right) d \varsigma & \leq \int_{0}^{T} \frac{\widetilde{\Lambda}_{2} d s}{1+\widetilde{\lambda}_{p q}(T-\varsigma)^{\beta}} \\
& \leq \frac{\widetilde{\Lambda}_{2}}{\widetilde{\lambda}_{p q}} \int_{0}^{T} \frac{d \varsigma}{(T-\varsigma)^{\beta}}=\frac{1}{\widetilde{\lambda}_{p q}}\left(\frac{\widetilde{\Lambda}_{2} T^{1-\beta}}{1-\beta}\right)
\end{aligned}
$$

Next, because of inequality $(T-\varsigma)^{\beta} \leq T^{\beta}$ valid for any $\varsigma \in[0, T]$ and Lemma 2.3, one has

$$
\begin{aligned}
\int_{0}^{T} E_{\beta, 1}\left(-\tilde{\lambda}_{p q}(T-\varsigma)^{\beta}\right) d \varsigma & \geq \widetilde{\Lambda}_{1} \int_{0}^{T} \frac{d \varsigma}{1+\widetilde{\lambda}_{p q}(T-\varsigma)^{\beta}} \geq \frac{\tilde{\Lambda}_{1}}{\tilde{\lambda}_{p q}} \int_{0}^{T} \frac{d \varsigma}{\frac{1}{2}+(T-\varsigma)^{\beta}} \\
& \geq \frac{\widetilde{\Lambda}_{1}}{\widetilde{\lambda}_{p q}} \int_{0}^{T} \frac{d \varsigma}{\frac{1}{2}+T^{\beta}}=\frac{1}{\widetilde{\lambda}_{p q}}\left(\frac{2 \widetilde{\Lambda}_{1} T}{1+2 T^{\beta}}\right)
\end{aligned}
$$

Combining (2.9) and (2.8), we conclude that

$$
\frac{1}{\widetilde{\lambda}_{p q}}\left(\frac{\widetilde{\Lambda}_{1} 2 T}{1+2 T^{\beta}}\right) \leq \int_{0}^{T} E_{\beta, 1}\left(-\tilde{\lambda}_{p q}(T-\varsigma)^{\beta}\right) d \varsigma \leq \frac{1}{\widetilde{\lambda}_{p q}}\left(\frac{\widetilde{\Lambda}_{2} T^{1-\beta}}{1-\beta}\right) .
$$

Denoting $\tilde{M}_{\beta}^{\dagger}\left(\tilde{\Lambda}_{1}\right)=\left(\frac{\tilde{\Lambda}_{1} 2 T}{1+2 T^{\beta}}\right), \tilde{M}_{\beta}^{\dagger \dagger}\left(\widetilde{\Lambda}_{2}\right)=\left(\frac{\tilde{\Lambda}_{2} T^{1-\beta}}{1-\beta}\right)$ shows that $(2.6)$ holds.

Lemma 2.6 Suppose that there exist positive constants such that $m_{0} \leq\left|\Phi^{\varepsilon}(t)\right| \leq M_{0}$, $\forall t \in[0, T]$. By choosing $\varepsilon \in\left(0, \frac{m_{0}}{2}\right)$, we have

$$
\frac{m_{0}}{2} \leq|\Phi(t)| \leq \mathcal{P}\left(m_{0}, M_{0}\right)
$$

where $\mathcal{P}\left(m_{0}, M_{0}\right)=M_{0}+\frac{m_{0}}{2}$.

Proof First of all, we have

$$
\begin{aligned}
\left|\Phi^{\varepsilon}(t)\right| & \leq|\Phi(t)|+\left|\Phi^{\varepsilon}(t)-\Phi(t)\right| \leq|\Phi(t)|+\sup _{t \in[0, T]}\left|\Phi^{\varepsilon}(t)-\Phi(t)\right| \\
& \leq|\Phi(t)|+\left\|\Phi^{\varepsilon}-\Phi\right\|_{\mathcal{L}^{\infty}(0, T)} \leq|\Phi(t)|+\varepsilon .
\end{aligned}
$$

From (2.12), we know that

$$
|\Phi(t)| \geq\left|\Phi^{\varepsilon}(t)\right|-\varepsilon \geq m_{0}-\varepsilon \geq \frac{m_{0}}{2}
$$

Similarly, we get

$$
|\Phi(t)| \leq M_{0}+\varepsilon<M_{0}+\frac{m_{0}}{2} .
$$

Denoting $\mathcal{P}\left(m_{0}, M_{0}\right)=M_{0}+\frac{m_{0}}{2}$, and combining (2.13) with (2.14) leads to (2.11).

Lemma 2.7 Let $\Phi:[0, T] \rightarrow \mathbb{R}^{+}$be a continuous function, then we have

$$
\frac{m_{0} \tilde{M}_{\beta}^{\dagger}\left(\widetilde{\Lambda}_{1}\right)}{2 \tilde{\lambda}_{p q}} \leq \int_{0}^{T} E_{\beta, 1}\left(-\tilde{\lambda}_{p q}(T-\varsigma)^{\beta}\right) \Phi(\varsigma) d \varsigma \leq \frac{\mathcal{P}\left(m_{0}, M_{0}\right) \tilde{M}_{\beta}^{\dagger \dagger}\left(\tilde{\Lambda}_{2}\right)}{\tilde{\lambda}_{p q}}
$$




\subsection{The formula of source term $f$}

In this section, we introduce the mild solution of the following initial value problem:

$$
\left\{\begin{array}{l}
\partial_{t} u(x, y, t)=\partial_{t}^{1-\beta}\left(u_{x x}(x, y, t)+u_{y y}(x, y, t)\right)+\mathbf{H}(x, y, t) \\
\quad(x, y, t) \in \Omega \times(0, T) \\
u(x, y, t)=0, \quad(x, y) \in \partial \Omega, t \in(0, T] \\
u(x, y, 0)=a(x, y), \quad(x, y) \in \Omega .
\end{array}\right.
$$

We use the separation of variables to obtain the solution of (2.16). Suppose that the exact $u$ is defined by Fourier series

$$
u(x, y, t)=\sum_{p=1}^{\infty} \sum_{q=1}^{\infty} u_{p q}(t) \mathcal{S}_{p q}(x, y)
$$

in which $\mathcal{S}_{p q}(x, y)=\sin (p x) \sin (q y)$. Taking the Laplace transform of (2.16) we obtain that

$$
\varsigma \widetilde{u_{p q}}(\varsigma)-\widetilde{a_{p q}}=-\tilde{\lambda}_{p q} \varsigma^{1-\beta} \widetilde{u_{p q}}(\varsigma)+\widetilde{\mathbf{H}_{p q}}(\varsigma), \quad \Re(\varsigma)>0
$$

in which $\widetilde{v}$ is the Laplace transform of function $v$, and so

$$
\widetilde{u_{p q}}(s)=\frac{\varsigma^{\beta-1}}{\varsigma^{\beta}+\widetilde{\lambda}_{p q}} \widetilde{a_{p q}}+\frac{\varsigma^{\beta-1}}{\varsigma^{\beta}+\widetilde{\lambda}_{p q}} \widetilde{\mathbf{H}_{p q}}(\varsigma)
$$

where $\mathbf{H}_{p q}(t)=\left\langle\mathbf{H}(\cdot, \cdot, t), \mathcal{S}_{p q}(x, y)\right\rangle$. It follows from Lemma 2.3 and the uniqueness of Laplace transform that

$$
u_{p q}(t)=E_{\beta, 1}\left(-\tilde{\lambda}_{p q} t^{\beta}\right) a_{p q}+\int_{0}^{t} E_{\beta, 1}\left(-\tilde{\lambda}_{p q}(t-\varsigma)^{\beta}\right) \mathbf{H}_{p q}(\varsigma) d \varsigma
$$

By letting $t=T$ in the last equality, recalling $a_{p q}=0$ and $\mathbf{H}_{p q}(\varsigma)=\Phi(\varsigma) f_{p q}$, we have

$$
u_{p q}(T)=g_{p q}=f_{p q} \int_{0}^{T} E_{\beta, 1}\left(-\tilde{\lambda}_{p q}(T-\varsigma)^{\beta}\right) \Phi(\varsigma) d \varsigma .
$$

We get the formula of the source function $f$ as

$$
f(x, y)=\sum_{p=1}^{\infty} \sum_{q=1}^{\infty} \frac{g_{p q} \mathcal{S}_{p q}(x, y)}{\int_{0}^{T} E_{\beta, 1}\left(-\tilde{\lambda}_{p q}(T-\varsigma)^{\beta}\right) \Phi(\varsigma) d \varsigma}
$$

where

$$
\begin{aligned}
& a_{p q}=\frac{4}{\pi^{2}} \int_{0}^{\pi} \int_{0}^{\pi} a(x, y) \mathcal{S}_{p q}(x, y) d x d y, \\
& g_{p q}=\frac{4}{\pi^{2}} \int_{0}^{\pi} \int_{0}^{\pi} g(x, y) \mathcal{S}_{p q}(x, y) d x d y, \\
& \mathbf{H}_{p q}(s)=\frac{4}{\pi^{2}} \int_{0}^{\pi} \int_{0}^{\pi} \mathbf{H}(x, y, s) \mathcal{S}_{p q}(x, y) d x d y,
\end{aligned}
$$

and where we note that $E_{\beta, 1}\left(-\tilde{\lambda}_{p q}(T-\varsigma)^{\beta}\right)>0$ and $\Phi(\varsigma)>0$ for $0 \leq \varsigma \leq T$. 
Theorem 2.1 Let $\Phi:[0, T] \rightarrow \mathbb{R}$ be as in Lemma 2.7. Then the solution $(u(x, y, t), f(x, y))$ of Problem (1.1) is unique.

Proof Let $f_{1}$ and $f_{2}$ be the source functions corresponding to the final values $g_{1}$ and $g_{2}$, respectively. Suppose that $g_{1}=g_{2}$. Then we prove that $f_{1}=f_{2}$. Using the fact that $E_{\beta, 1}\left(-\tilde{\lambda}_{p q}(T-\varsigma)^{\beta}\right) \geq 0$ for $\varsigma \leq T$. Using Lemma 2.2, we have

$$
\begin{aligned}
\int_{0}^{T} E_{\beta, 1}\left(-\widetilde{\lambda}_{p q}(T-\varsigma)^{\beta}\right) \Phi(\varsigma) d \varsigma & \geq \frac{m_{0}}{2} \int_{0}^{T} E_{\beta, 1}\left(-\tilde{\lambda}_{p q}(T-\varsigma)^{\beta}\right) d \varsigma \\
& =\frac{m_{0}}{2} T E_{\beta, 2}\left(-\tilde{\lambda}_{p q} T^{\beta}\right)>0 .
\end{aligned}
$$

Therefore, we get

$$
f_{1}(x, y)-f_{2}(x, y)=\sum_{p=1}^{\infty} \sum_{q=1}^{\infty} \frac{\left(g_{p q}^{1}-g_{p q}^{2}\right) \mathcal{S}_{p q}(x, y)}{\int_{0}^{T} E_{\beta, 1}\left(-\widetilde{\lambda}_{p q}(T-\varsigma)^{\beta}\right) \Phi(\varsigma) d \varsigma}=0 .
$$

The proof is complete.

Theorem 2.2 The inverse source problem is ill-posed.

Proof To illustrate the ill-posedness of our problem, the relevant counterexample is indicated. Choose the input final data $g_{m n}(x, y)=\frac{\mathcal{S}_{m n}(x, y)}{\sqrt{m n}}$. By (2.22), the source term corresponding to $g_{m n}$ is

$$
\begin{aligned}
f_{m n}(x, y) & =\sum_{p=1}^{\infty} \sum_{q=1}^{\infty} \frac{g_{m n} \mathcal{S}_{p q}(x, y)}{\int_{0}^{T} E_{\beta, 1}\left(-\widetilde{\lambda}_{p q}(T-\varsigma)^{\beta}\right) \Phi(\varsigma) d \varsigma} \\
& =\sum_{p=1}^{\infty} \sum_{q=1}^{\infty} \frac{\frac{\mathcal{S}_{m n}(x, y)}{\sqrt{m n}} \mathcal{S}_{p q}(x, y)}{\int_{0}^{T} E_{\beta, 1}\left(-\widetilde{\lambda}_{p q}(T-\varsigma)^{\beta}\right) \Phi(\varsigma) d \varsigma} \\
& =\frac{\mathcal{S}_{m n}(x, y)}{\sqrt{m n}} \int_{0}^{T} E_{\beta, 1}\left(-\widetilde{\lambda}_{m n}(T-\varsigma)^{\beta}\right) \Phi(\varsigma) d \varsigma .
\end{aligned}
$$

Assume that input final data $g(x, y)=0$. By (2.22), the source term corresponding to $g$ is $f(x, y)=0$. The error in $\mathcal{L}^{2}(\Omega)$ norm between two input final data is

$$
\left\|g_{m n}-g\right\|_{\mathcal{L}^{2}(\Omega)}=\left\|\frac{\mathcal{S}_{m n}(x, y)}{\sqrt{m n}}\right\|_{\mathcal{L}^{2}(\Omega)}=\frac{1}{\sqrt{m n}} \rightarrow 0 \quad \text { when } m, n \rightarrow \infty
$$

The error in $\mathcal{L}^{2}$ norm between two corresponding source terms is

$$
\begin{aligned}
\left\|f_{m n}-f\right\|_{\mathcal{L}^{2}(\Omega)}^{2} & =\left(\frac{\pi^{2}}{4}\right)\left\|\frac{\mathcal{S}_{m n}(x, y)}{\sqrt{m n} \int_{0}^{T} E_{\beta, 1}\left(-\widetilde{\lambda}_{m n}(T-\varsigma)^{\beta}\right) \Phi(\varsigma) d \varsigma}\right\|_{\mathcal{L}^{2}(\Omega)}^{2} \\
& =\left(\frac{\pi^{2}}{4}\right) \frac{1}{m n}\left(\int_{0}^{T} E_{\beta, 1}\left(-\widetilde{\lambda}_{m n}(T-\varsigma)^{\beta}\right) \Phi(\varsigma) d \varsigma\right)^{-2} \\
& \geq\left(\frac{\pi^{2}}{4}\right) \frac{1}{m n} \frac{\left(\int_{0}^{T} E_{\beta, 1}\left(-\widetilde{\lambda}_{m n}(T-\varsigma)^{\beta}\right) d \varsigma\right)^{-2}}{\left|\mathcal{P}\left(m_{0}, M_{0}\right)\right|}
\end{aligned}
$$




$$
=\left(\frac{\pi^{2}}{4}\right) \frac{1}{\mathcal{P}\left(m_{0}, M_{0}\right) \widetilde{M}_{\beta}^{\dagger \dagger}\left(\widetilde{\Lambda}_{2}\right)} \frac{\tilde{\lambda}_{m n}^{2}}{m n} \rightarrow \infty \quad \text { as } m, n \rightarrow \infty .
$$

Hence, from (2.27) and (2.28), there is in general no stability result. Thus, problem (1.1) is ill-posed in the Hadamard sense in $\mathcal{L}^{2}$ norm.

\subsection{Conditional stability of source term $f$}

Theorem 2.3 For $\mathcal{M}>0$ such that $\|f\|_{H^{\delta}(\Omega)} \leq \mathcal{M}$,

$$
\|f\|_{\mathcal{L}^{2}(\Omega)} \leq \widetilde{C}(\delta, \mathcal{M})\|g\|_{\mathcal{L}^{2}(\Omega)}^{\frac{\delta}{\delta+1}}
$$

in which

$$
\widetilde{C}(\delta, \mathcal{M})=\left(\frac{2}{m_{0}\left(\widetilde{M}_{\beta}^{\dagger}\left(\widetilde{\Lambda}_{1}\right)\right)}\right)^{\frac{\delta}{\delta+1}} \mathcal{M}^{\frac{1}{\delta+1}} .
$$

Proof From (2.22), combining Hölder inequality and Lemma 2.7, we obtain

$$
\begin{aligned}
\|f\|_{\mathcal{L}^{2}(\Omega)}^{2} & \leq\left(\sum_{p=1}^{\infty} \sum_{q=1}^{\infty} \frac{\left|\left\langle g_{p q}, \mathcal{S}_{p q}(x, y)\right\rangle\right|^{2}}{\left|\int_{0}^{T} E_{\beta, 1}\left(-\widetilde{\lambda}_{p q}(T-\varsigma)^{\beta}\right) \Phi(\varsigma) d \varsigma\right|^{2 \delta+2}}\right)^{\frac{1}{\delta+1}}\left(\sum_{p=1}^{\infty} \sum_{q=1}^{\infty}|| g_{p q},\left.\mathcal{S}_{p q}(x, y)\right|^{2}\right)^{\frac{\delta}{\delta+1}} \\
& \leq\left(\sum_{p=1}^{\infty} \sum_{q=1}^{\infty} \frac{\left|\left\langle f_{p q}, \mathcal{S}_{p q}(x, y)\right\rangle\right|^{2}}{\left|\int_{0}^{T} E_{\beta, 1}\left(-\widetilde{\lambda}_{p q}(T-\varsigma)^{\beta}\right) \Phi(\varsigma) d \varsigma\right|^{2 \delta}}\right)^{\frac{1}{\delta+1}}\|g\|_{\mathcal{L}^{2}(\Omega)}^{\frac{2 \delta}{\delta+1}} \\
& \leq\left(\frac{\sum_{p=1}^{\infty} \sum_{q=1}^{\infty} \widetilde{\lambda}_{p q}^{2 \delta}\left|\left\langle f_{p q}, \mathcal{S}_{p q}(x, y)\right\rangle\right|^{2}}{\left|\frac{m_{0}}{2}\right|^{2 \delta}\left(\widetilde{M}_{\beta}^{\dagger}\left(\widetilde{\Lambda}_{1}\right)\right)^{2 \delta}}\right)^{\frac{1}{\delta+1}}\|g\|_{\mathcal{L}^{2}(\Omega)}^{\frac{2 \delta}{\delta+1}} .
\end{aligned}
$$

From (2.31), we conclude that

$$
\|f\|_{\mathcal{L}^{2}(\Omega)}^{2} \leq\left(\frac{2}{\left|m_{0}\right|\left(\widetilde{M}_{\beta}^{\dagger}\left(\widetilde{\Lambda}_{1}\right)\right)}\right)^{\frac{2 \delta}{\delta+1}}\|f\|_{H^{\delta}(\Omega)}^{\frac{2}{\delta+1}}\|g\|_{\mathcal{L}^{2}(\Omega)}^{\frac{2 \delta}{\delta+1}} .
$$

\section{Fourier truncation regularization and error estimate}

In this section, we eliminate all the components of large $p, q$ from the solution where the positive integer $\mathcal{N}_{\mathcal{T} \mathcal{R}}$ plays the role of the regularization parameter. The nature of our regularization method is just eliminating all high frequencies from the solution and considering instead of (2.22) only for $\tilde{\lambda}_{p q}=p^{2}+q^{2}$, where $\mathcal{N}_{\mathcal{T} \mathcal{R}}$ is a suitable positive constant. We note that the $\mathcal{N}_{\mathcal{T} \mathcal{R}}$ constant will be selected appropriately as a formal parameter. This regularization method is quite simple and convenient to handle some of the problems posed. The current article is devoted to establishing such an approach for problem (1.1). Let

$$
\begin{aligned}
& \bar{B}_{\varepsilon}:=\left\{p, q \in \mathbb{N}^{*}, \tilde{\lambda}_{p q}=p^{2}+q^{2} \leq \mathcal{N}_{\mathcal{T} \mathcal{R}}\right\}, \\
& \underline{B}_{\varepsilon}:=\left\{p, q \in \mathbb{N}^{*}, \tilde{\lambda}_{p q}=p^{2}+q^{2}>\mathcal{N}_{\mathcal{T} \mathcal{R}}\right\} .
\end{aligned}
$$


We have the regularized solution as follows:

$$
f_{\varepsilon}^{\mathcal{N} \mathcal{T} \mathcal{R}}(x, y)=\sum_{p, q \in \bar{B}_{\varepsilon}} \frac{g_{p q}^{\varepsilon} \mathcal{S}_{p q}(x, y)}{\int_{0}^{T} E_{\beta, 1}\left(-\widetilde{\lambda}_{p q}(T-\varsigma)^{\beta}\right) \Phi^{\varepsilon}(\varsigma) d \varsigma} .
$$

Next, we show error estimation for $\left\|f(x, y)-f_{\varepsilon}^{\mathcal{N}_{\mathcal{T}} \mathcal{R}}(x, y)\right\|_{\mathcal{L}^{2}(\Omega)}$ and give convergence rate under a suitable choice for the regularization parameter.

\subsection{Convergence estimate under an a priori regularization parameter choice rule}

Theorem 3.1 Let $f_{\varepsilon}^{\mathcal{N} \mathcal{T}_{\mathcal{R}}}(x, y)$ be the regularized solution of Problem (1.1) with observed data $g^{\varepsilon}(x, y)$ and let $f(x, y)$ be the exact solution of Problem (1.1) with exact data $g(x, y)$. By choosing parameter regularization $\mathcal{N}_{\mathcal{T} \mathcal{R}}=[\zeta]$, where $[\zeta]$ denotes the largest integer less than or equal to $\zeta$,

- If $0<\delta \leq 1$ then, by choosing $\zeta=\left(\frac{\mathcal{M}}{\varepsilon}\right)^{\frac{1}{\delta+1}}$, we obtain

$$
\left\|f(x, y)-f_{\mathcal{\varepsilon}}^{\mathcal{N}} \mathcal{T}_{\mathcal{R}}(x, y)\right\|_{\mathcal{L}^{2}(\Omega)} \leq \varepsilon^{\frac{\delta}{\delta+1}} \mathcal{M}^{\frac{1}{\delta+1}} \mathcal{D}\left(m_{0}, \beta, f, \Lambda_{1}\right)
$$

- If $\delta>1$, then, by choosing $\zeta=\left(\frac{\mathcal{M}}{\varepsilon}\right)^{\frac{1}{2}}$, we obtain

$$
\left\|f(x, y)-f_{\varepsilon}^{\mathcal{N}} \mathcal{T R}(x, y)\right\|_{\mathcal{L}^{2}(\Omega)} \leq \varepsilon^{\frac{1}{2}} \mathcal{M}^{\frac{1}{2}} \mathcal{D}\left(m_{0}, \beta, f, \Lambda_{1}\right)
$$

where $\mathcal{D}\left(m_{0}, \beta, f, \Lambda_{1}\right)=\frac{\pi}{2}\left(1+\max \left\{\frac{1}{\left|m_{0}\right| \widetilde{\mathcal{M}}_{\beta}^{\dagger}\left(\Lambda_{1}\right)}, \frac{\|f\|_{\mathcal{L}^{2}(\Omega)}}{\left|m_{0}\right|}\right\}\right)$

Proof of Theorem 3.1 Using (2.22) and (3.3) and the triangle inequality, we have

$$
\begin{array}{r}
f(x, y)-f_{\varepsilon}^{\mathcal{N}_{\mathcal{T}}(x, y)} \\
=\sum_{p=1}^{\infty} \sum_{q=1}^{\infty} \frac{g_{p q} \mathcal{S}_{p q}(x, y)}{\int_{0}^{T} E_{\beta, 1}\left(-\widetilde{\lambda}_{p q}(T-\varsigma)^{\beta}\right) \Phi(\varsigma) d \varsigma} \\
\quad-\sum_{p, q \in \bar{B}_{\varepsilon}} \frac{g_{p q}^{\varepsilon} \mathcal{S}_{p q}(x, y)}{\int_{0}^{T} E_{\beta, 1}\left(-\widetilde{\lambda}_{p q}(T-\varsigma)^{\beta}\right) \Phi^{\varepsilon}(\varsigma) d \varsigma} \\
=\sum_{p=1}^{\infty} \sum_{q=1}^{\infty} \frac{g_{p q} \mathcal{S}_{p q}(x, y)}{\int_{0}^{T} E_{\beta, 1}\left(-\widetilde{\lambda}_{p q}(T-\varsigma)^{\beta}\right) \Phi(\varsigma) d \varsigma} \\
\quad-\sum_{p, q \in \bar{B}_{\varepsilon}} \frac{g_{p q} \mathcal{S}_{p q}(x, y)}{\int_{0}^{T} E_{\beta, 1}\left(-\tilde{\lambda}_{p q}(T-\varsigma)^{\beta}\right) \Phi(\varsigma) d \varsigma} \\
+\sum_{p, q \in \bar{B}_{\varepsilon}} \frac{g_{p q} \mathcal{S}_{p q}(x, y)}{\int_{0}^{T} E_{\beta, 1}\left(-\widetilde{\lambda}_{p q}(T-\varsigma)^{\beta}\right) \Phi(s) d s} \\
\quad-\sum_{p, q \in \bar{B}_{\varepsilon}} \frac{g_{p q}^{\varepsilon} \mathcal{S}_{p q}(x, y)}{\int_{0}^{T} E_{\beta, 1}\left(-\widetilde{\lambda}_{p q}(T-\varsigma)^{\beta}\right) \Phi^{\varepsilon}(\varsigma) d \varsigma} .
\end{array}
$$

Using (3.6), we can write

$$
f(x, y)-f_{\varepsilon}^{\mathcal{N} \mathcal{T} \mathcal{R}}(x, y)
$$




$$
\begin{aligned}
& =\underbrace{\sum_{p, q \in \underline{B}_{\varepsilon}} \frac{g_{p q} \mathcal{S}_{p q}(x, y)}{\int_{0}^{T} E_{\beta, 1}\left(-\widetilde{\lambda}_{p q}(T-\varsigma)^{\beta}\right) \Phi(\varsigma) d \varsigma}}_{:=\mathcal{A}_{1}}+\underbrace{\sum_{p, q \in \bar{B}_{\varepsilon}} \frac{\left(g_{p q}(x)-g_{p q}^{\varepsilon}(x)\right) \mathcal{S}_{p q}(x, y)}{\int_{0}^{T} E_{\beta, 1}\left(-\widetilde{\lambda}_{p q}(T-\varsigma)^{\beta}\right) \Phi^{\varepsilon}(\varsigma) d \varsigma}}_{:=\mathcal{A}_{2}} \\
& +\underbrace{\sum_{p, q \in \bar{B}_{\varepsilon}} \frac{g_{p q} \mathcal{S}_{p q}(x, y)}{\int_{0}^{T} E_{\beta, 1}\left(-\widetilde{\lambda}_{p q}(T-\varsigma)^{\beta}\right) \Phi(\varsigma) d \varsigma} \times \sum_{p, q \in \bar{B}_{\varepsilon}} \frac{\int_{0}^{T} E_{\beta, 1}\left(-\tilde{\lambda}_{p q}(T-\varsigma)^{\beta}\right)\left(\Phi^{\varepsilon}(\varsigma)-\Phi(\varsigma)\right) d \varsigma}{\int_{0}^{T} E_{\beta, 1}\left(-\widetilde{\lambda}_{p q}(T-\varsigma)^{\beta}\right) \Phi^{\varepsilon}(\varsigma) d \varsigma}}_{:=\mathcal{A}_{3}} .
\end{aligned}
$$

Step 1 . Firstly, we have the following estimate:

$$
\begin{aligned}
\left\|\mathcal{A}_{1}\right\|_{\mathcal{L}^{2}(\Omega)}^{2} & =\frac{\pi^{2}}{4} \sum_{p, q \in \underline{B}_{\varepsilon}} \frac{\left|\left\langle g_{p q}, \mathcal{S}_{p q}(x, y)\right\rangle\right|^{2}}{\left|\int_{0}^{T} E_{\beta, 1}\left(-\widetilde{\lambda}_{p q}(T-\varsigma)^{\beta}\right) \Phi(\varsigma) d \varsigma\right|^{2}} \\
& =\frac{\pi^{2}}{4} \sum_{p, q \in \underline{B}_{\varepsilon}}\left|\left\langle f_{p q}, \mathcal{S}_{p q}(x, y)\right\rangle\right|^{2} \\
& \leq \frac{\pi^{2}}{4} \sum_{p, q \in \underline{B}_{\varepsilon}}\left(1+\widetilde{\lambda}_{p q}\right)^{-2 \delta}\left(1+\tilde{\lambda}_{p q}\right)^{2 \delta}\left|\left\langle f_{p q}, \mathcal{S}_{p q}(x, y)\right\rangle\right|^{2} \\
& \leq \frac{\pi^{2}}{4}\left(1+\mathcal{N}_{\mathcal{T} \mathcal{R}}\right)^{-2 \delta} \mathcal{M}^{2} .
\end{aligned}
$$

Hence, we obtain

$$
\left\|\mathcal{A}_{1}\right\|_{\mathcal{L}^{2}(\Omega)} \leq \frac{\pi}{2}\left(1+\mathcal{N}_{\mathcal{T} \mathcal{R}}\right)^{-\delta} \mathcal{M}
$$

Step 2 . The term $\left\|\mathcal{A}_{2}\right\|_{\mathcal{L}^{2}(\Omega)}$ is bounded by

$$
\begin{aligned}
\left\|\mathcal{A}_{2}\right\|_{\mathcal{L}^{2}(\Omega)}^{2} & \leq \frac{\pi^{2}}{4} \sum_{p, q \in \bar{B}_{\varepsilon}} \frac{\left|g_{p q}(x)-g_{p q}^{\varepsilon}(x)\right|^{2}}{\left|\int_{0}^{T} E_{\beta, 1}\left(-\widetilde{\lambda}_{p q}(T-\varsigma)^{\beta}\right) \Phi^{\varepsilon}(\varsigma) d \varsigma\right|^{2}} \\
& \leq \frac{\pi^{2}}{4} \sum_{p, q \in \bar{B}_{\varepsilon}} \frac{\widetilde{\lambda}_{p q}^{2}\left|g_{p q}-g_{p q}^{\varepsilon}\right|^{2}}{\left|m_{0}\right|^{2}\left(\widetilde{\mathcal{M}}_{\beta}^{\dagger}\left(\widetilde{\Lambda}_{1}\right)\right)^{2}} \\
& \leq \frac{\pi^{2}}{4} \sup _{1 \leq p \leq \mathcal{N}_{\mathcal{T} \mathcal{R}}} \sup _{1 \leq q \leq \mathcal{N}_{\mathcal{T} \mathcal{R}}} \frac{\widetilde{\lambda}_{p q}^{2}}{\left|m_{0}\right|^{2}\left(\widetilde{\mathcal{M}}_{\beta}^{\dagger}\left(\widetilde{\Lambda}_{1}\right)\right)^{2}} \sum_{p, q \in \bar{B}_{\varepsilon}}\left|g_{p q}-g_{p q}^{\varepsilon}\right|^{2} \\
& \leq \frac{\pi^{2} \widetilde{\lambda}_{p q}^{2}}{4\left|m_{0}\right|^{2}\left(\widetilde{\mathcal{M}}_{\beta}^{\dagger}\left(\Lambda_{1}\right)\right)^{2}}\left\|g-g^{\varepsilon}\right\|_{\mathcal{L}^{2}(\Omega)}^{2} \leq \frac{\pi^{2} \mathcal{N}_{\mathcal{T} \mathcal{R}^{2} \varepsilon^{2}}}{4\left|m_{0}\right|^{2}\left(\widetilde{\mathcal{M}}_{\beta}^{\dagger}\left(\Lambda_{1}\right)\right)^{2}} .
\end{aligned}
$$

Therefore,

$$
\left\|\mathcal{A}_{2}\right\|_{\mathcal{L}(\Omega)} \leq \frac{\varepsilon \pi \mathcal{N}_{\mathcal{T} \mathcal{R}}}{2\left|m_{0}\right| \widetilde{\mathcal{M}}_{\beta}^{\dagger}\left(\Lambda_{1}\right)}
$$

Step 3 . The term $\left\|\mathcal{A}_{3}\right\|_{\mathcal{L}^{2}(\Omega)}$ can be estimated as follows:

$$
\left\|\mathcal{A}_{3}\right\|_{\mathcal{L}^{2}(\Omega)}^{2} \leq \frac{\pi^{2}}{4}\left(\sum_{p, q \in \bar{B}_{\varepsilon}}\left|\frac{\left|\left\langle g_{p q}, \mathcal{S}_{p q}(x, y)\right\rangle\right|}{\int_{0}^{T} E_{\beta, 1}\left(-\widetilde{\lambda}_{p q}(T-\varsigma)^{\beta}\right) \Phi^{\varepsilon}(\varsigma) d \varsigma}\right|^{2}\right)
$$




$$
\times\left(\sum_{p, q \in \bar{B}_{\varepsilon}}\left|\frac{\int_{0}^{T} E_{\beta, 1}\left(-\tilde{\lambda}_{p q}(T-\varsigma)^{\beta}\right)\left(\Phi^{\varepsilon}(\varsigma)-\Phi(\varsigma)\right) d \varsigma}{\int_{0}^{T} E_{\beta, 1}\left(-\widetilde{\lambda}_{p q}(T-\varsigma)^{\beta}\right) \Phi^{\varepsilon}(\varsigma) d \varsigma}\right|^{2}\right) .
$$

From (3.12), we get

$$
\begin{aligned}
\left\|\mathcal{A}_{3}\right\|_{\mathcal{L}^{2}(\Omega)}^{2} & \leq \frac{\pi^{2}}{4}\left(\sum_{p, q \in \bar{B}_{\varepsilon}} \frac{\left|\int_{0}^{T} E_{\beta, 1}\left(-\widetilde{\lambda}_{p q}(T-\varsigma)^{\beta}\right)\left(\Phi^{\varepsilon}(\varsigma)-\Phi(\varsigma)\right) d \varsigma\right|^{2}}{\left|\int_{0}^{T} E_{\beta, 1}\left(-\widetilde{\lambda}_{p q}(T-\varsigma)^{\beta}\right) \Phi^{\varepsilon}(\varsigma) d \varsigma\right|^{2}}\right) \\
& \times\left(\sum_{p, q \in \bar{B}_{\varepsilon}} \frac{\left|\left\langle g_{p q}, \mathcal{S}_{p q}(x, y)\right\rangle\right|^{2}}{\left|\int_{0}^{T} E_{\beta, 1}\left(-\widetilde{\lambda}_{p q}(T-\varsigma)^{\beta}\right) \Phi(\varsigma) d \varsigma\right|^{2}}\right) \\
\leq & \frac{\pi^{2}}{4} \frac{\left\|\Phi^{\varepsilon}-\Phi\right\|_{\mathcal{L}^{\infty}(0, T)}^{2}}{\left|m_{0}\right|^{2}} \sum_{p=1}^{\infty} \sum_{q=1}^{\infty} \frac{\left|\left\langle g_{p q}, \mathcal{S}_{p q}(x, y)\right\rangle\right|^{2}}{\left|\int_{0}^{T} E_{\beta, 1}\left(-\widetilde{\lambda}_{p q}(T-\varsigma)^{\beta}\right) \Phi(\varsigma) d \varsigma\right|^{2}} \\
= & \frac{\pi^{2}}{4} \frac{\left\|\Phi^{\varepsilon}-\Phi\right\|_{\mathcal{L}^{\infty}(0, T)}^{2}}{\left|m_{0}\right|^{2}}\|\|_{\mathcal{L}^{2}(\Omega)}^{2} \leq \frac{\pi^{2} \varepsilon^{2} \mid f \|_{\mathcal{L}^{2}(\Omega)}^{2}}{4\left|m_{0}\right|^{2}} .
\end{aligned}
$$

From (3.12), we conclude that

$$
\left\|\mathcal{A}_{3}\right\|_{\mathcal{L}^{2}(\Omega)} \leq \frac{\pi \varepsilon\|f\|_{\mathcal{L}^{2}(\Omega)}}{2\left|m_{0}\right|}
$$

Combining (3.8), (3.11), and (3.12) yields

$$
\begin{aligned}
& \| f(x, y)-f_{\varepsilon}^{\mathcal{N}_{\mathcal{T} \mathcal{R}}(x, y) \|_{\mathcal{L}^{2}(\Omega)}} \\
& \quad \leq \frac{\pi \mathcal{M}}{2\left(1+\mathcal{N}_{\mathcal{T} \mathcal{R})^{\delta}}\right.}+\frac{\pi \varepsilon \mathcal{N}_{\mathcal{T} \mathcal{R}}}{2\left|m_{0}\right| \widetilde{\mathcal{M}}_{\beta}^{\dagger}\left(\Lambda_{1}\right)}+\frac{\pi \varepsilon\|f\|_{\mathcal{L}^{2}(\Omega)}}{2\left|m_{0}\right|} .
\end{aligned}
$$

Using the fact that $\mathcal{N}_{\mathcal{T} \mathcal{R}} \leq \zeta \leq \mathcal{N}_{\mathcal{T R}}+1$ gives

$$
\begin{aligned}
& \left\|f(x, y)-f_{\varepsilon}^{\mathcal{N} \mathcal{T} \mathcal{R}}(x, y)\right\|_{\mathcal{L}^{2}(\Omega)} \\
& \quad \leq \frac{\pi}{2}\left[\zeta^{-\delta} \mathcal{M}+\varepsilon \zeta \max \left\{\frac{1}{\left|m_{0}\right| \widetilde{\mathcal{M}}_{\beta}^{\dagger}\left(\Lambda_{1}\right)}, \frac{\|f\|_{\mathcal{L}^{2}(\Omega)}}{\left|m_{0}\right|}\right\}\right] \\
& \leq \varepsilon^{\frac{\delta}{\delta+1}} \mathcal{M}^{\frac{1}{\delta+1}} \frac{\pi}{2}\left(1+\max \left\{\frac{1}{\left|m_{0}\right| \widetilde{\mathcal{M}}_{\beta}^{\dagger}\left(\Lambda_{1}\right)}, \frac{\|f\|_{\mathcal{L}^{2}(\Omega)}}{\left|m_{0}\right|}\right\}\right) .
\end{aligned}
$$

\subsection{Convergence estimate under an a posteriori regularization parameter choice rule}

In this subsection, by using the discrepancy principle, we consider an a posteriori regularization parameter choice. Define

$$
\mathcal{R}_{\mathcal{N}_{\mathcal{T} \mathcal{R}}} g^{\varepsilon}=\sum_{p, q \in \bar{B}_{\varepsilon}} g_{p q}^{\varepsilon} \mathcal{S}_{p q}(x, y)
$$

Because of the discrepancy principle, we take $\mathbb{K}=\mathbb{K}\left(\varepsilon, g^{\varepsilon}\right)$ as the solution of

$$
\left\|\left(I-\mathcal{R}_{\mathcal{N}_{\mathcal{T} \mathcal{R}}}\right) g^{\varepsilon}\right\|_{\mathcal{L}^{2}(\Omega)} \leq \tau \varepsilon \leq \|\left(I-\mathcal{R}_{\left.\mathcal{N}_{\mathcal{T} \mathcal{R}^{-1}}\right) g^{\varepsilon}} \|_{\mathcal{L}^{2}(\Omega)}, \quad \tau>1\right.
$$


For this choice rule, we get an upper bound estimate for the $\mathcal{N}_{\mathcal{T} \mathcal{R}}$ in the following lemma.

Lemma 3.1 We have

$$
\mathcal{N}_{\mathcal{T} \mathcal{R}} \leq\left(\frac{\pi \widetilde{\mathcal{M}}_{\beta}^{\dagger \dagger}\left(\widetilde{\Lambda}_{2}\right) \mathcal{P}\left(m_{0}, M_{0}\right) \mathcal{M}}{2(\tau-1) \varepsilon}\right)^{\frac{1}{1+\delta}}
$$

Proof From $\left\|g^{\varepsilon}-g\right\|_{\mathcal{L}^{2}(\Omega)} \leq \varepsilon$ and (3.19), we have

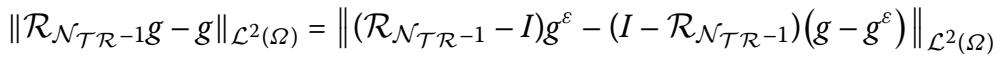

$$
\begin{aligned}
& \geq \|\left(\mathcal { R } _ { \mathcal { N } _ { \mathcal { T } \mathcal { R } ^ { - 1 } } - I ) g ^ { \varepsilon } } \| _ { \mathcal { L } ^ { 2 } ( \Omega ) } - \| \left(I-\mathcal{R}_{\left.\mathcal{N}_{\mathcal{T} \mathcal{R}^{-1}}\right)\left(g-g^{\varepsilon}\right)} \|_{\mathcal{L}^{2}(\Omega)}\right.\right. \\
& \geq(\tau-1) \varepsilon \text {. }
\end{aligned}
$$

On the other hand, for $\tilde{\lambda}_{p q} \geq \mathcal{N}_{\mathcal{T} \mathcal{R}}$, we obtain

$$
\begin{aligned}
& \int_{0}^{T} E_{\beta, 1}\left(-\tilde{\lambda}_{p q}(T-\varsigma)^{\beta}\right) \Phi(\varsigma) d \varsigma \\
& \quad \leq\left|\mathcal{P}\left(m_{0}, M_{0}\right)\right| \int_{0}^{T} E_{\beta, 1}\left(-\widetilde{\lambda}_{p q}(T-\varsigma)^{\beta}\right) \Phi(\varsigma) d \varsigma \\
& \quad=\left|\mathcal{P}\left(m_{0}, M_{0}\right)\right| \frac{\widetilde{\mathcal{M}}_{\beta}^{\dagger \dagger}\left(\tilde{\Lambda}_{2}\right)}{\widetilde{\lambda}_{p q}} \leq \frac{\left|\mathcal{P}\left(m_{0}, M_{0}\right)\right| \widetilde{\mathcal{M}}_{\beta}^{\dagger \dagger}\left(\widetilde{\Lambda}_{2}\right)}{\mathcal{N}_{\mathcal{T} \mathcal{R}}} .
\end{aligned}
$$

This implies that

$$
\begin{aligned}
& \left\|\mathcal{R}_{\mathcal{N}_{\mathcal{T}^{-1}} g-g}\right\|_{\mathcal{L}^{2}(\Omega)}^{2} \\
& =\frac{\pi^{2}}{4} \sum_{p, q \in \underline{B}_{\varepsilon}}\left|\left\langle g(\cdot, \cdot), \mathcal{S}_{p q}(x, y)\right\rangle\right|^{2} \\
& =\frac{\pi^{2}}{4} \sum_{p, q \in \underline{B}_{\varepsilon}}\left|\int_{0}^{T} E_{\beta, 1}\left(-\tilde{\lambda}_{p q}(T-\varsigma)^{\beta}\right) \Phi(\varsigma) d \varsigma\left\langle f_{p q}, \mathcal{S}_{p q}(x, y)\right\rangle\right|^{2} \\
& \leq \frac{\pi^{2}}{4} \frac{\left|\mathcal{P}\left(m_{0}, M_{0}\right)\right|^{2}\left(\widetilde{\mathcal{M}}_{\beta}^{\dagger \dagger}\left(\tilde{\Lambda}_{2}\right)\right)^{2}}{\left(\mathcal{N}_{\mathcal{T} \mathcal{R}}\right)^{2}} \sum_{p, q \in \underline{B}_{\varepsilon}}\left|\left\langle f_{p q}, \mathcal{S}_{p q}(x, y)\right\rangle\right|^{2} \\
& \leq \frac{\pi^{2}}{4} \frac{\left|\mathcal{P}\left(m_{0}, M_{0}\right)\right|^{2}\left(\widetilde{\mathcal{M}}_{\beta}^{\dagger \dagger}\left(\tilde{\Lambda}_{2}\right)\right)^{2}}{\left(\mathcal{N}_{\mathcal{T} \mathcal{R}}\right)^{2}} \sum_{p, q \in \underline{B}_{\varepsilon}}\left(1+\tilde{\lambda}_{p q}\right)^{-2 \delta}\left(1+\tilde{\lambda}_{p q}\right)^{2 \delta}\left|\left\langle f_{p q}, \mathcal{S}_{p q}(x, y)\right\rangle\right|^{2} \\
& \leq \frac{\pi^{2}}{4} \frac{\left|\mathcal{P}\left(m_{0}, M_{0}\right)\right|^{2}\left(\widetilde{\mathcal{M}}_{\beta}^{\dagger \dagger}\left(\widetilde{\Lambda}_{2}\right)\right)^{2}}{\left(\mathcal{N}_{\mathcal{T} \mathcal{R}}\right)^{2}\left(\mathcal{N}_{\mathcal{T} \mathcal{R}}\right)^{2 \delta}} \sum_{p, q \in \underline{B}_{\varepsilon}}\left(1+\tilde{\lambda}_{p q}\right)^{2 \delta}\left|\left\langle f_{p q}, \mathcal{S}_{p q}(x, y)\right)\right|^{2} \\
& \leq \frac{\pi^{2}}{4} \frac{\left|\mathcal{P}\left(m_{0}, M_{0}\right)\right|^{2}\left(\widetilde{\mathcal{M}}_{\beta}^{\dagger \dagger}\left(\tilde{\Lambda}_{2}\right)\right)^{2}\|f\|_{H^{\delta}(\Omega)}^{2}}{\left(\mathcal{N}_{\mathcal{T} \mathcal{R}}\right)^{2+2 \delta}} \\
& \leq \frac{\pi^{2}}{4} \frac{\left|\mathcal{P}\left(m_{0}, M_{0}\right)\right|^{2}\left(\widetilde{\mathcal{M}}_{\beta}^{\dagger \dagger}\left(\tilde{\Lambda}_{2}\right)\right)^{2} \mathcal{M}^{2}}{\left(\mathcal{N}_{\mathcal{T} \mathcal{R}}\right)^{2+2 \delta}} .
\end{aligned}
$$


Hence, we conclude that

$$
\left\|F_{\mathcal{N}-1} g-g\right\|_{\mathcal{L}^{2}(\Omega)} \leq \frac{\pi\left|\mathcal{P}\left(m_{0}, M_{0}\right)\right| \widetilde{\mathcal{M}}_{\beta}^{\dagger \dagger}\left(\widetilde{\Lambda}_{2}\right) \mathcal{M}}{2\left(\mathcal{N}_{\mathcal{T} \mathcal{R}}\right)^{1+\delta}}
$$

Combining (3.21) and (3.24), we conclude that

$$
(\tau-1) \varepsilon \leq \frac{\pi\left|\mathcal{P}\left(m_{0}, M_{0}\right)\right| \widetilde{\mathcal{M}}_{\beta}^{\dagger \dagger}\left(\widetilde{\Lambda}_{2}\right) \mathcal{M}}{2\left(\mathcal{N}_{\mathcal{T} \mathcal{R}}\right)^{1+\delta}}
$$

From (3.25), so we can obtain

$$
\mathcal{N}_{\mathcal{T} \mathcal{R}} \leq\left(\frac{\pi\left|\mathcal{P}\left(m_{0}, M_{0}\right)\right| \widetilde{\mathcal{M}}_{\beta}^{\dagger \dagger}\left(\widetilde{\Lambda}_{2}\right) \mathcal{M}}{2(\tau-1) \varepsilon}\right)^{\frac{1}{1+\delta}} .
$$

Next we present an error estimate for the approximate solution of problem (1.1).

Theorem 3.2 $\operatorname{Let}_{\varepsilon}^{\mathcal{N}_{\mathcal{T}} \mathcal{R}}$ and f be as in Theorem 3.1. Then we obtain

$$
\left\|f(x, y)-f_{\varepsilon}^{\mathcal{N}_{\mathcal{T} \mathcal{R}}}(x, y)\right\|_{\mathcal{L}^{2}(\Omega)} \leq \varepsilon^{\frac{\delta}{\delta+1}} \mathcal{M}^{\frac{1}{\delta+1}}\left[Q_{1}+Q_{2}(\tau+1)^{\frac{\delta}{\delta+1}}\right]
$$

where

$$
\begin{aligned}
Q_{1} & =\left(\frac{\pi \widetilde{\mathcal{M}}_{\beta}^{\dagger \dagger}\left(\tilde{\Lambda}_{2}\right)\left|\mathcal{P}\left(m_{0}, M_{0}\right)\right|}{2(\tau-1)\left|m_{0}\right|^{\delta+1}}\right)^{\frac{1}{\delta+1}} \max \left\{\|f\|_{\mathcal{L}^{2}(\Omega)}, \frac{\pi}{2 \widetilde{M}_{\beta}^{\dagger}\left(\widetilde{\Lambda}_{1}\right)}\right\}, \\
Q_{2} & =\frac{1}{\left|\frac{m_{0}}{2}\right|^{\frac{\delta}{\delta+1}}\left(\tilde{M}_{\beta}^{\dagger}\left(\tilde{\Lambda}_{1}\right)\right)^{\frac{\delta}{\delta+1}}} .
\end{aligned}
$$

Proof Using the triangle inequality, we have

$$
\begin{aligned}
& \left\|f(x, y)-f_{\varepsilon}^{\mathcal{N}_{\mathcal{T} \mathcal{R}}}(x, y)\right\|_{\mathcal{L}^{2}(\Omega)}
\end{aligned}
$$

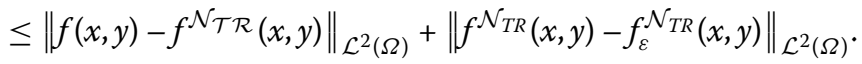

We split the proof into two steps.

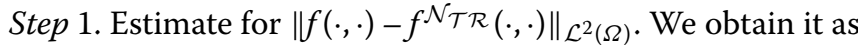

$$
\begin{aligned}
& \| f(x, y)-f^{\mathcal{N}_{\mathcal{T} \mathcal{R}}(x, y) \|_{H^{\delta}(\Omega)}} \\
& \leq\left\|\sum_{p=\mathcal{N}_{\mathcal{T} \mathcal{R}^{+1}}}^{\infty} \sum_{q=\mathcal{N}_{\mathcal{T} \mathcal{R}^{+1}}}^{\infty}\left\langle f_{p q}, \mathcal{S}_{p q}(x, y)\right\rangle\right\| \\
& \quad=\left(\sum_{p=\mathcal{N}_{\mathcal{T} \mathcal{R}}+1}^{\infty} \sum_{q=\mathcal{N}_{\mathcal{T} \mathcal{R}}+1}^{\infty}\left(1+\tilde{\lambda}_{p q}\right)^{2 \delta}\left|\left\langle f(\cdot, \cdot), \mathcal{S}_{p q}(x, y)\right\rangle\right|^{2}\right)^{1 / 2} \leq \mathcal{M} .
\end{aligned}
$$

From (3.19), we get

$$
\left\|A f(x, y)-A f^{\mathcal{N}_{\mathcal{T} \mathcal{R}}(x, y)}\right\|_{\mathcal{L}^{2}(\Omega)}
$$




$$
\begin{aligned}
& \leq\left\|\left(I-\mathcal{R}_{\mathcal{N}_{\mathcal{T} \mathcal{R}}}\right) g\right\|_{\mathcal{L}^{2}(\Omega)} \\
& \leq\left\|\left(I-\mathcal{R}_{\left.N_{T R}\right)}\right) g^{\epsilon}+\left(I-\mathcal{R}_{\mathcal{N}_{\mathcal{T} \mathcal{R}}}\right)\left(g-g^{\epsilon}\right)\right\|_{\mathcal{L}^{2}(\Omega)} \\
& \leq\left\|\left(I-\mathcal{R}_{\mathcal{N}_{\mathcal{T} \mathcal{R}}}\right) g^{\varepsilon}\right\|_{\mathcal{L}^{2}(\Omega)}+\left\|\left(I-\mathcal{R}_{\mathcal{N}_{\mathcal{T}}}\right)\left(g-g^{\varepsilon}\right)\right\|_{\mathcal{L}^{2}(\Omega)} \\
& \leq(\tau+1) \varepsilon .
\end{aligned}
$$

Therefore, we have

$$
\left\|f(\cdot, \cdot)-f^{\mathcal{N}} \mathcal{T} \mathcal{R}_{(\cdot, \cdot)}\right\|_{\mathcal{L}^{2}(\Omega)} \leq Q_{2}((\tau+1) \varepsilon)^{\frac{\delta}{\delta+1}} .
$$

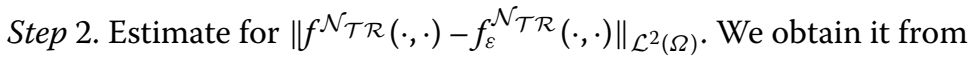

$$
\begin{aligned}
& f^{\mathcal{N}_{\mathcal{T} \mathcal{R}}}(x, y)-f_{\varepsilon}^{\mathcal{N}_{\mathcal{T} \mathcal{R}}}(x, y) \\
& =\sum_{p, q \in \bar{B}_{\varepsilon}} \frac{g_{p q} \mathcal{S}_{p q}(x, y)}{\int_{0}^{T} E_{\beta, 1}\left(-\widetilde{\lambda}_{p q}(T-\varsigma)^{\beta}\right) \Phi(\varsigma) d \varsigma-\sum_{p, q \in \bar{B}_{\varepsilon}} \frac{g_{p q}^{\varepsilon} \mathcal{S}_{p q}(x, y)}{\int_{0}^{T} E_{\beta, 1}\left(\tilde{\lambda}_{p q}(T-\varsigma)^{\beta}\right) \Phi^{\varepsilon}(\varsigma) d \varsigma}} \\
& \leq \sum_{p, q \in \bar{B}_{\varepsilon}} \frac{g_{p q} \mathcal{S}_{p q}(x, y)}{\int_{0}^{T} E_{\beta, 1}\left(-\widetilde{\lambda}_{p q}(T-\varsigma)^{\beta}\right) \Phi(\varsigma) d \varsigma}-\sum_{p, q \in \bar{B}_{\varepsilon}} \frac{g_{p q} \mathcal{S}_{p q}(x, y)}{\int_{0}^{T} E_{\beta, 1}\left(-\widetilde{\lambda}_{p q}(T-\varsigma)^{\beta}\right) \Phi^{\varepsilon}(\varsigma) d \varsigma} \\
& +\sum_{p, q \in \bar{B}_{\varepsilon}} \frac{\left(g_{p q}-g_{p q}^{\varepsilon}\right) \mathcal{S}_{p q}(x, y)}{\int_{0}^{T} E_{\beta, 1}\left(-\widetilde{\lambda}_{p q}(T-\varsigma)^{\beta}\right) \Phi^{\varepsilon}(\varsigma) d \varsigma} .
\end{aligned}
$$

From (3.33), we know that

$$
\begin{aligned}
f^{\mathcal{N}} \mathcal{N}_{\mathcal{R}}(x, y)-f_{\varepsilon}^{\mathcal{N}_{\mathcal{T}}}(x, y) & \\
\leq & \underbrace{\sum_{p, q \in \bar{B}_{\varepsilon}} \frac{\int_{0}^{T} E_{\beta, 1}\left(-\widetilde{\lambda}_{p q}(T-\varsigma)^{\beta}\right)\left(\Phi(\varsigma)-\Phi^{\varepsilon}(\varsigma)\right) d \varsigma}{\int_{0}^{T} E_{\beta, 1}\left(-\widetilde{\lambda}_{p q}(T-\varsigma)^{\beta}\right) \Phi^{\varepsilon}(\varsigma) d \varsigma} \times \sum_{p, q \in \bar{B}_{\varepsilon}} \frac{g_{p q} \mathcal{S}_{p q}(x, y)}{\int_{0}^{T} E_{\beta, 1}\left(-\widetilde{\lambda}_{p q}(T-\varsigma)^{\beta}\right) \Phi(\varsigma) d \varsigma}}_{:=\mathcal{A}_{3}} \\
& +\underbrace{\sum_{p, q \in \bar{B}_{\varepsilon}} \frac{\left\langle g_{p q}-g_{p q}^{\varepsilon}, \mathcal{S}_{p q}(x, y)\right\rangle}{\int_{0}^{T} E_{\beta, 1}\left(-\widetilde{\lambda}_{p q}(T-\varsigma)^{\beta}\right) \Phi^{\varepsilon}(\varsigma) d \varsigma}}_{:=\mathcal{A}_{4}} .
\end{aligned}
$$

From (3.33), it is easy to check that

$$
\left\|\mathcal{A}_{3}\right\|_{\mathcal{L}^{2}(\Omega)} \leq \frac{\varepsilon\|f\|_{\mathcal{L}^{2}(\Omega)}}{m_{0}}
$$

Our estimate of $\mathcal{A}_{4}$ is based on Lemma 2.7, and we obtain

$$
\begin{aligned}
\left\|\mathcal{A}_{4}\right\|_{\mathcal{L}^{2}(\Omega)}^{2} & =\frac{\pi^{2}}{4} \sum_{p, q \in \bar{B}_{\varepsilon}}\left|\frac{\left\langle g_{p q}(x, y)-g_{p q}^{\varepsilon}(x, y), \mathcal{S}_{p q}(x, y)\right\rangle}{\int_{0}^{T} E_{\beta, 1}\left(-\widetilde{\lambda}_{p q}(T-\varsigma)^{\beta}\right) \Phi^{\varepsilon}(\varsigma) d \varsigma}\right|^{2} \\
& \leq \frac{\pi^{2}}{4} \sum_{p, q \in \bar{B}_{\varepsilon}} \frac{\widetilde{\lambda}_{p q}^{2}\left|\left\langle g_{p q}(x, y)-g_{p q}^{\varepsilon}(x, y), \mathcal{S}_{p q}(x, y)\right\rangle\right|^{2}}{\left|m_{0}\right|^{2}\left(\widetilde{M}_{\beta}^{\dagger}\left(\widetilde{\Lambda}_{1}\right)\right)^{2}}
\end{aligned}
$$




$$
\leq \frac{\pi^{2}}{4} \frac{\varepsilon^{2}\left(\mathcal{N}_{\mathcal{T} \mathcal{R}}\right)^{2}}{m_{0}^{2}\left(\widetilde{M}_{\beta}^{\dagger}\left(\widetilde{\Lambda}_{1}\right)\right)^{2}}
$$

Hence, we get

$$
\left\|\mathcal{A}_{4}\right\|_{\mathcal{L}^{2}(\Omega)} \leq \frac{\pi \varepsilon \mathcal{N}_{\mathcal{T} \mathcal{R}}}{2 m_{0} \widetilde{M}_{\beta}^{\dagger}\left(\widetilde{\Lambda}_{1}\right)}
$$

From the above observations, we deduce that

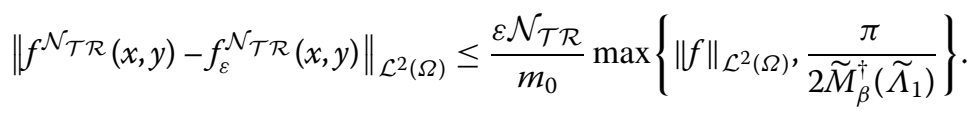

Substituting (3.26) into (3.38), we obtain

$$
\left\|f^{\mathcal{N} \mathcal{T} \mathcal{R}}(x, y)-f_{\mathcal{E}}^{\mathcal{N}} \mathcal{T R}(x, y)\right\|_{\mathcal{L}^{2}(\Omega)} \leq \varepsilon^{\frac{\delta}{\delta+1}} \mathcal{M}^{\frac{1}{\delta+1}} Q_{1} .
$$

Combining Steps 1 and 2, we obtain the final estimate as follows:

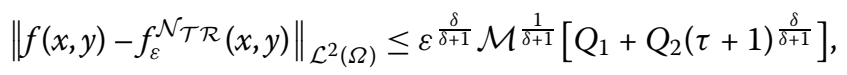

in which $Q_{1}$ depends on $m_{0}, M_{0}, \Lambda_{1}, \Lambda_{2}, \delta, f, \tau, \beta$, and $Q_{2}$ depends on $m_{0}, \delta, \beta, \Lambda_{1}$ defined in (3.28). This completes the proof.

\section{Acknowledgements}

The authors would like to thank the kindness of the Editor and Reviewers in helping improve the manuscript.

\section{Funding}

Not applicable.

\section{Availability of data and materials}

Not applicable.

\section{Competing interests}

The authors declare that they have no competing interests.

\section{Authors' contributions}

The authors declare that the study was realized in collaboration with the same responsibility. All authors read and approved the final manuscript. All authors contributed equally to the writing of this paper.

\section{Author details}

'Institute of Research and Development, Duy Tan University, Da Nang, Vietnam. ${ }^{2}$ Department of Mathematics, Cankaya University, Ankara, Turkey. ${ }^{3}$ Department of Medical Research, China Medical University Hospital, China Medical University, Taichung, Taiwan. ${ }^{4}$ Institute of Space Sciences, Magurele-Bucharest, Romania. ${ }^{5}$ Department of Mathematics and Computer Science, University of Science, Ho Chi Minh City, Vietnam. ${ }^{6}$ Vietnam National University, Ho Chi Minh City, Vietnam. ${ }^{7}$ Applied Analysis Research Group, Faculty of Mathematics and Statistics, Ton Duc Thang University, Ho Chi Minh City, Vietnam.

\section{Publisher's Note}

Springer Nature remains neutral with regard to jurisdictional claims in published maps and institutional affiliations.

Received: 1 November 2019 Accepted: 17 February 2020 Published online: 27 February 2020

\section{References}

1. Barkai, E., Metzler, R., Klafter, J.: From continuous time random walks to the fractional Fokker-Planck equation. Phys. Rev. E 61(1), 132 (2000)

2. Gorenflo, R., Mainardi, F: Some recent advances in theory and simulation of fractional diffusion processes. J. Comput. Appl. Math. 229(2), 400-415 (2009)

3. Podlubny, I.: Fractional Differential Equations. Academic Press, London (1999) 
4. Raberto, M., Scalas, E., Mainardi, F.: Waiting-times and returns in high-frequency financial data: an empirical study. Physica A 314(1), 749-755 (2002)

5. Yong, Z: Basic Theory of Fractional Differential Equations. World Scientific, Singapore (2014)

6. Schneider, W.R., Wyss, W.: Fractional diffusion and wave equations. J. Math. Phys. 30(1), 134-144 (1989)

7. Hirata, H., Miao, C.: Space-time estimates of linear flow and application to some nonlinear integro-differential equations corresponding to fractional-order time derivative. Adv. Differ. Equ. 2, 217-236 (2002)

8. Kochubei, A.N.: Cauchy problem for fractional diffusion-wave equations with variable coefficients. Appl. Anal. 93(10), 2211-2242 (2014)

9. Carillo, S., Valente, V., Caffarelli, G.V.: A linear viscoelasticity problem with a singular memory kernel: an existence and uniqueness result. Differ. Integral Equ. 26(9-10), 1115-1125 (2013)

10. Carillo, S., Valente, V., Caffarelli, G.V.: Heat conduction with memory: a singular kernel problem. Evol. Equ. Control Theory 3(3), 399-410 (2014)

11. Yong, Z., He, J.W., Ahmad, B., Nguyen, H.T.: Existence and regularity results of a backward problem for fractional diffusion equations. Math. Methods Appl. Sci. 42(18), 6775-6790 (2019)

12. Badia, A.E., Duong, T.H.: On an inverse source problem for the heat equation. Application to a pollution detection problem. J. Inverse III-Posed Probl. 10, 585-599 (2002)

13. Isakov, V.: Inverse Problems for Partial Differential Equations. Applied Mathematical Sciences, vol. 127. Springer, New York (1998)

14. Badia, A.E., Hajj, A.E.: Identification of dislocations in materials from boundary measurements. SIAM J. Appl. Math. 73 84-103 (2013)

15. Anastasio, M.A., Zhang, J., Modgil, D., Rivière, P.J.L.: Application of inverse source concepts to photoacoustic tomography. Inverse Probl. 23, S21-S35 (2007)

16. Stefanov, P., Uhlmann, G.: Thermoacoustic tomography with variable sound speed. Inverse Probl. 25, 075011 (2009)

17. Hämäläinen, M., Hari, R., Ilmoniemi, R.J., Knuutila, J., Lounasmaa, O.V.: Magnetoencephalography-theory, instrumentation, and applications to noninvasive studies of the working human brain. Rev. Mod. Phys. 65, 413-497 (1993)

18. Mosher, J.C., Lewis, P.S., Leahy, R.M.: Multiple dipole modeling and localization from spatio-temporal MEG data. IEEE Trans. Biomed. Eng. 39, 541-557 (1992)

19. Arridge, S.R.: Optical tomography in medical imaging. Inverse Probl. 15, R41-R93 (1999)

20. Nguyen, H.T., Le, D.L., Nguyen, V.T.: Regularized solution of an inverse source problem for a time fractional diffusion equation. Appl. Math. Model. 40(19-20), 8244-8264 (2016)

21. Wei, T., Wang, J: A modified quasi-boundary value method for an inverse source problem of the time-fractional diffusion equation. Appl. Numer. Math. 78, 95-111 (2014)

22. Wang, J.G., Zhou, A.S., Wei, T.: Two regularization methods to identify a space-dependent source for the time-fractional diffusion equation. Appl. Numer. Math. 68, 39-57 (2013)

23. Zhang, Z.Q., Wei, T.: Identifying an unknown source in time-fractional diffusion equation by a truncation method Appl. Math. Comput. 219(11), 5972-5983 (2013)

24. Kirane, M., Malik, A.S., Al-Gwaiz, M.A.: An inverse source problem for a two dimensional time fractional diffusion equation with nonlocal boundary conditions. Math. Methods Appl. Sci. 36(9), 1056-1069 (2013)

25. Kirane, M., Malik, A.S.: Determination of an unknown source term and the temperature distribution for the linear heat equation involving fractional derivative in time. Appl. Math. Comput. 218(1), 163-170 (2011)

26. Sakamoto, K., Yamamoto, M.: Initial value/boundary value problems for fractional diffusion-wave equations and applications to some inverse problems. J. Math. Anal. Appl. 382, 426-447 (2011)

\section{Submit your manuscript to a SpringerOpen ${ }^{\circ}$ journal and benefit from:}

- Convenient online submission

- Rigorous peer review

- Open access: articles freely available online

- High visibility within the field

- Retaining the copyright to your article

Submit your next manuscript at $\gg$ springeropen.com 